Musées, Patrimoine et Culture scientifiques et techniques

$153 \mid 2014$

mai-juin 2014

\title{
Le chantier de rénovation de l'herbier national
}

\section{Pascale Joannot}

URL : http://journals.openedition.org/ocim/1387

DOI : $10.4000 /$ ocim. 1387

ISSN : 2108-646X

Éditeur

OCIM

Édition imprimée

Date de publication : 25 juin 2014

ISSN : 0994-1908

\section{Référence électronique}

Pascale Joannot, « Le chantier de rénovation de l'herbier national », La Lettre de I'OCIM [En ligne] 153 | 2014, mis en ligne le 25 juin 2016, consulté le 19 avril 2019. URL : http:// journals.openedition.org/ocim/1387 ; DOI : 10.4000/ocim.1387 


\section{Le chantier de rénovation de l'herbier national}

\section{Pascale Joannot}

Directrice adjointe des collections, en charge des chantiers de rénovation au Muséum national d'Histoire naturelle

Le Muséum national d'Histoire naturelle, qui a la garde de près de 65 millions de spécimens, a décidé, en octobre 2000, de redonner à la conservation et à la gestion de ses collections, ainsi qu'à la diffusion des connaissances qui s'y rattachent, la place qu'elles méritent et a ouvert un chantier de rénovation des collections, dont l'objectif est de mener à bien la réorganisation structurelle, fonctionnelle et spatiale des collections. Tout en créant une direction des collections et en prenant un règlement intérieur des collections, des chantiers de conservation, d'inventaire et informatisation des collections ont été organisés. Le plus important d'entre eux est celui de la botanique, achevé, après 4 ans de travaux, en décembre 2012.

\section{Le constat}

Depuis la création des collections de botanique en 1650, l'herbier national est devenu le plus important du monde. Le bâtiment de $10365 \mathrm{~m}^{2}$, construit en 1936 avec l'aide de la Fondation Rockefeller, conçu pour accueillir 6 millions de spécimens, s'est, face à l'accroissement des fonds, révélé trop petit, peu fonctionnel et inadapté à la conservation.

Deux solutions ont été envisagées : déplacer l'herbier dans un bâtiment neuf ou rénover l'existant pour doubler sa capacité d'accueil et le mettre aux normes de conservation. Cette dernière solution a été retenue dès 2002. Le programme de rénovation de l'herbier validé en 2006 et lancé en 2009 comprenait deux volets : la rénovation du bâtiment et le chantier des collections de l'herbier national.

\section{Le projet}

La rédaction du programme a été le fruit d'un travail d'équipe constituée par la direction de la rénovation du Muséum, la direction des collections et des botanistes, aidés par un consultant extérieur, Gilles Pacaud.

Mener à bien la rénovation du bâtiment, classé Monument Historique, était pour le bureau d'étude SNC Lavalin et le cabinet d'architecture GAP Studio, complexe car il fallait travailler en site occupé. L'aménagement intérieur a été repensé pour faciliter l’organisation

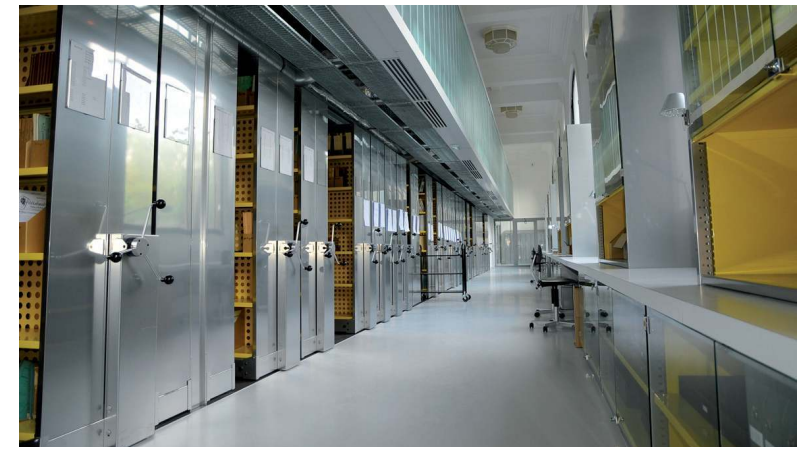

Les nouveaux rayonnages mobiles pour le rangement de l'herbier (c) MNHN/ Françoise Bouazzat

et le travail des botanistes. Après avoir consulté d'autres institutions, nous avons opté pour un traitement de l'air contrôlé (température $19^{\circ} \mathrm{C}$ - humidité relative $50 \%)$ dans les réserves de collections.

Le cheminement d'une plante arrivée du terrain jusqu'à sa mise en collection a été prévu : local d'arrivée, chambre froide pour la décontamination, atelier de préparation des spécimens, local d'informatisation, circulation, mise en collection, salle de consultation.

La bibliothèque de botanique aménagée au rez-de-chaussée a également été prévue.

\section{Le chantier des collections}

En parallèle du chantier bâtiment, la direction des collections a piloté le chantier du traitement des collections botaniques. Nous avions pour objectifs d'intégrer dans la collection des planches d'herbier non attachées ; de reconditionner des spécimens ; de numériser l'ensemble de la collection et de reclasser les planches selon l'ordre systématique en vigueur, et non plus par ordre géographique comme c'était le cas.

Pour mener à bien ce chantier nous avons eu recours à des prestataires de services. Nous avons lancé des appels d'offres et donc rédigé en amont une étape essentielle : le cahier des charges. Ce travail d'équipe pluridisciplinaire, rigoureux et précis n'est pas simple lorsque les masses à traiter sont si importantes. 


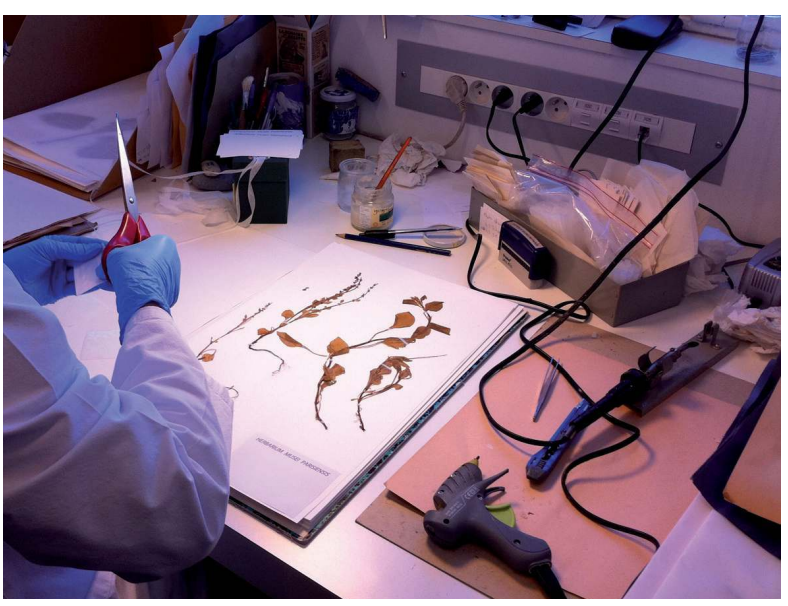

Une planche d'herbier en cours d'attachage (c) Pascale Joannot

\section{Le chantier dit « d'attachage »}

Dans la collection de botanique, des spécimens étaient en attente d'être montés ou « attachés », c'est-à-dire retirés de leur conditionnement d'origine (une feuille de papier journal où ils avaient séchés), puis posés sur une planche de papier fort, fixés par de la colle ou des bandelettes de papier gommé. La plante attachée n'a de valeur scientifique que si sa " carte d'identité » l'accompagne : étiquette comprenant le nom de la plante, du collecteur, du lieu de collecte et la date.

Le rez-de-chaussée du bâtiment de botanique a été aménagé pour recevoir les équipes de la Société Grahal, employant jusqu'à 22 personnes simultanément. Entre 2008 et 2012, 1460000 spécimens ont été attachés et classés. Les équipes ont effectué le pré-classement des planches, par famille botanique, pour faciliter leur intégration ultérieure dans la collection. Ces planches ont intégré la chaîne de numérisation.

\section{Le chantier de reconditionnement,} numérisation, reclassement et rangement des collections portait sur plusieurs opérations :

- le reconditionnement des planches : remplacement des chemises cartonnées regroupant plusieurs spécimens et des feuilles dites de papier sulfurisé protégeant chaque spécimen ;

- la numérisation : prise de photographies numériques des spécimens réalisée sur des chaînes automatisées et précédée par l'apposition d'une étiquette code barre sur chaque spécimen ;

- le reclassement : pour répondre aux besoins de la recherche, les botanistes du Muséum ont modifié l'organisation initiale par secteur géographique et ordonné les planches selon l'ordre taxonomique utilisant la

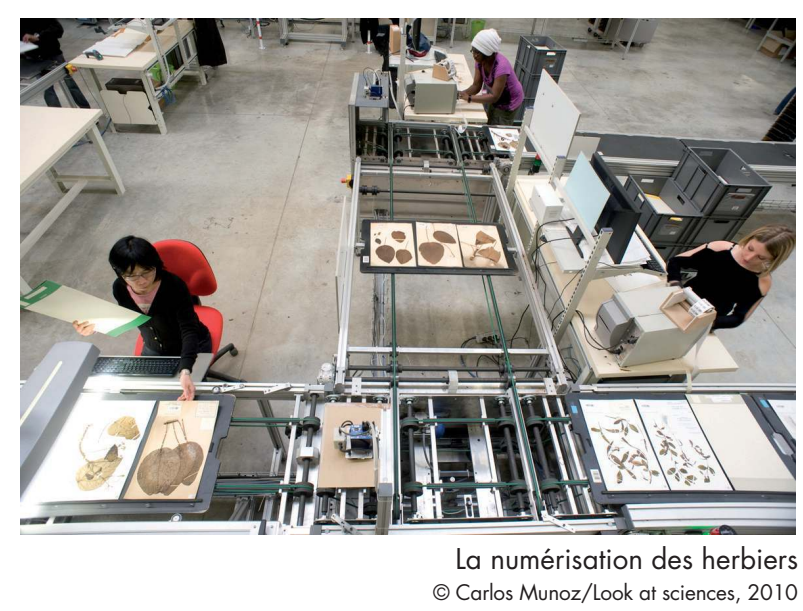

classification moderne des plantes dite APG3 (classification phylogénétique, troisième version de la classification botanique des angiospermes établie par l'A $n$ giosperms Phylogeny Group) le secteur géographique devenant la clé de rangement secondaire ;

- le rangement des planches : les planches traitées, stockées selon l'ordre taxonomique, ont été rangées dans les compactus, selon un plan d'occupation défini par les botanistes.

Ces opérations confiées à la société OBS ont été réalisées de mi 2010 à fin 2012. OBS a numérisé, sur un site extérieur au Muséum, 5528997 planches d'herbier. Des prestations de déménagement des collections, de fournitures et de stockage informatique ont complété ce chantier.

\section{Le résultat}

Ce chantier a permis de résorber l'immense retard de classement et de numérisation des spécimens, de rendre accessible la collection ( 8 millions de spécimens dont 500000 types) ; de retrouver un bon niveau d'échanges, d'acquisitions et de consultation (plus de 150 scientifiques/an). Les photographies en ligne constituent un herbier virtuel consultable, limitant la manipulation des spécimens et donc améliorant leur conservation.

Entièrement piloté par les équipes du Muséum et financé par nos tutelles, ce chantier de rénovation a coûté 26,2 millions $€$ : 15 millions $€$ pour le bâtiment et 11,2 millions $€$ pour le chantier des collections.

Depuis 2013, une nouvelle étape est engagée : la création du premier site Internet de sciences participatives (http://lesherbonautes.mnhn.fr) consacré aux collections de l'Herbier national où chacun peut alimenter une base de données.

Enfin, depuis le 27 novembre 2013 le Muséum a ouvert au public une galerie de botanique. 